\title{
Noise correlations in shear flows
}

\author{
B. Eckhardt ${ }^{1, a}$ and R. Pandit ${ }^{2, b}$ \\ 1 Fachbereich Physik, Philipps-Universität Marburg, 35032 Marburg, Germany \\ 2 Centre for Condensed Matter Theory, Department of Physics, Indian Institute of Science, Bangalore, 560012 , India
}

Received 26 February 2002 / Received in final form 11 March 2003

Published online 20 June 2003 - (C) EDP Sciences, Società Italiana di Fisica, Springer-Verlag 2003

\begin{abstract}
We consider the effects of a shear on velocity fluctuations in a flow. The shear gives rise to a transient amplification that not only influences the amplitude of perturbations but also their time correlations. We show that, in the presence of white noise, time correlations of transversal velocity components are exponential and that correlations of the longitudinal components are exponential with an algebraic prefactor. Cross correlations between transversal and downstream components are strongly asymmetric and provide a clear indication of non-normal amplification. We suggest experimental tests of our predictions.
\end{abstract}

PACS. 47.27.-i Turbulent flows, convection, and heat transfer - 05.40.Ca Noise - 47.20.Ft Instability of shear flows

\section{Introduction}

There has been a resurgence of theoretical interest in shear-driven flows, ubiquitous both in nature and in the laboratory [1], because the linearization of the NavierStokes equation about a laminar shear profile gives rise to a non-normal linear operator. The eigenstates of this operator are not orthogonal so, even if the laminar profile is asymptotically stable, perturbations can grow as transients for a while before submitting eventually to viscous damping [1-5]. This effect occurs generically if non-normal linear operators arise during a linear-stability analysis and it has been discussed at length in various contexts [6], including the hydrodynamical one we concentrate on here.

Several authors have advocated the use of pseudospectra in problems involving non-normal operators $[4,7]$. A complementary approach is to characterise the behaviour of non-normal systems by studying their response to an externally imposed noise. Farrell and Ioannou $[8,9]$ showed that, in general, this results in an increased variance; they determined amplification rates and the perturbations that give rise to the largest amplitudes for shear flows [10]. Rather little has been said about temporal correlations in such systems; an important, early exception is the study by Onuki [11] whose emphasis is quite different from ours as we explain below. But the temporal relation between lift-up and other instabilities is at the heart of the steady cycle that Waleffe et al. propose as the main mechanism for sustaining turbulent fluctuations in shear flows [12-14]. The idea is that downstream vortices give rise to downstream streaks by non-normal amplification, that the streaks go unstable to vertical vortex formation

\footnotetext{
a e-mail: bruno.eckhardt@physik.uni-marburg.de

b Also at: Jawaharlal Nehru Centre for Advanced Scientific Research, Bangalore, India
}

and that finally these vertical vortices are folded over into downstream vortices by the linear shear profile.

It is our aim here to present results on correlations in linear, non-normal systems driven by white noise and to discuss their relevance to perturbed shear flows. Specifically, we will show that temporal cross correlations between a streak and a vortex component can provide unambiguous signs of lift-up and non-normal amplification.

The formalism we use to study the fluctuations is similar to Rapid Distortion Theory or RDT $[15,16]$. We assume that we have a prescribed strong shear and follow a perturbation in Fourier space, using Kelvin modes. In order to avoid technical difficulties related to advecting frames of reference or time-dependent wave vectors, we use perturbations without variation in the flow direction. Nevertheless, as we will discuss later on, there is numerical evidence that this does not significantly affect the main conclusion that we draw about cross-correlations.

The outline of the paper is as follows: In Section 2 we discuss a stochastic model with two degrees of freedom, with one vortex and one shear component, that highlights the non-normal coupling and the temporal correlations to be expected. We then turn in Section 3 to a full discussion of temporal correlations in the stochastically forced Navier-Stokes equation linearised about a laminar shear profile. Section 4 contains concluding remarks, suggestions for experimental tests of our predictions, and a discussion of the relation of our work to earlier studies.

\section{A simple model}

\subsection{Vortex-streak coupling in shear flows}

Consider a linear shear profile, $\mathbf{U}_{0}=S z \mathbf{e}_{x}$. Coordinates are chosen in the meteorological convention, with $x$ 


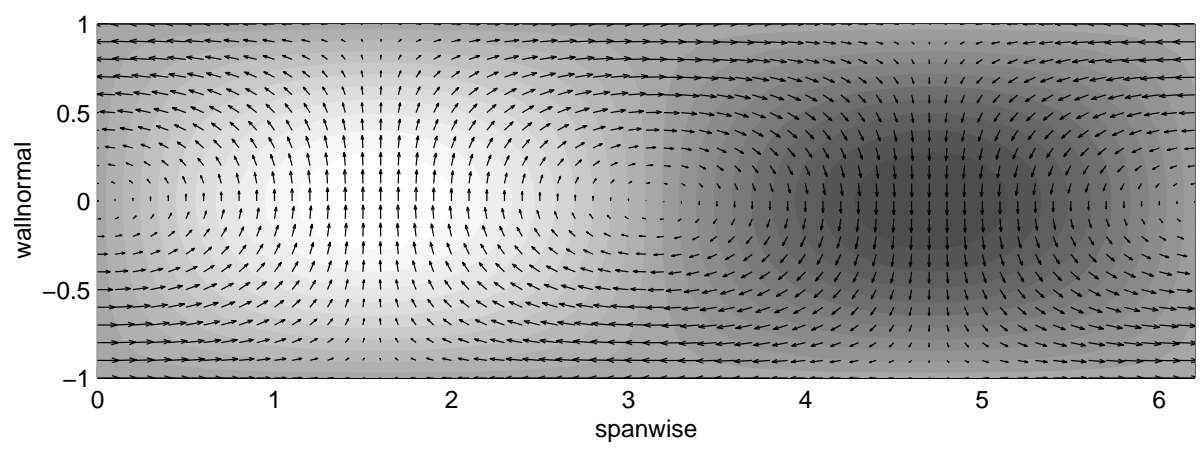

Fig. 1. Sketch of a vortex and a streak in a shear flow, looking downstream. The velocity components of the vortex lie in the plane and are indicated by the arrows. The streak has only one velocity component perpendicular to the plane; its values are indicated by a grey scale. The extrema of the streak (light and dark regions) are located near the maximal normal velocities. All scales of length and velocity are arbitrary.

pointing downstream, $y$ in the spanwise direction and $z$ pointing in the direction of the shear. The Navier-Stokes equation for the fluid velocity $\mathbf{u}$ linearised around this flow is

$$
\partial_{t} \mathbf{u}+(\mathbf{u} \cdot \nabla) \mathbf{U}_{0}+\left(\mathbf{U}_{0} \cdot \nabla\right) \mathbf{u}=-\nabla p+\nu \Delta \mathbf{u},
$$

where $p$ is the kinematic pressure and $\nu$ the kinematic viscosity of the fluid. To keep the analysis as simple as possible we work with the Fourier modes appropriate for periodic boundary conditions in spanwise and downstream directions, and free-slip boundary conditions on two parallel planes in the normal direction. The analysis of the linear problem with Kelvin modes [17-19] shows that modulations in the downstream direction give rise to a timedependent wave vector and faster-than-exponential damping. Farrell and Ioannou [10] also show that the most important modes for non-normal amplification do not have a downstream variation. Therefore, we consider only perturbations with wave numbers $\mathbf{k}=\left(0, k_{y}, k_{z}\right)$, where $k_{y}$ is continuous and $k_{z}=\pi n / d$, with $n$ an integer and $d$ the distance between the bounding planes. These considerations help us to identify scales for the flows considered in this section; their full significance will become clear in the next section.

In order to highlight the essentials of non-normal amplification, we now take a velocity field consisting of two modes, namely, a spanwise streak

$$
\mathbf{u}_{s}=\left(\begin{array}{c}
\alpha \sin \alpha y \cos \beta z \\
0 \\
0
\end{array}\right)
$$

and a downstream vortex

$$
\mathbf{u}_{\omega}=\left(\begin{array}{c}
0 \\
\beta \cos \alpha y \sin \beta z \\
-\alpha \sin \alpha y \cos \beta z
\end{array}\right)
$$

with amplitudes $s(t)$ and $\omega(t)$, i.e.,

$$
\mathbf{u}=s(t) \mathbf{u}_{s}+\omega(t) \mathbf{u}_{\omega}
$$

The two modes are sketched in Figure 1. In the linearised equation the pressure disappears as the velocity fields are divergence-free. The term $\left(\mathbf{U}_{0} \cdot \nabla\right) \mathbf{u}$ drops out and $(\mathbf{u} \cdot \nabla) \mathbf{U}_{0}$ results in a coupling between vortex and streak:

$$
\left(\begin{array}{l}
\dot{s} \\
\dot{\omega}
\end{array}\right)=\left(\begin{array}{cc}
-\nu\left(\alpha^{2}+\beta^{2}\right) & S \\
0 & -\nu\left(\alpha^{2}+\beta^{2}\right)
\end{array}\right)\left(\begin{array}{l}
s \\
\omega
\end{array}\right) .
$$

The matrix on the right hand side is not symmetric because of the coupling of both modes through the term $(\mathbf{u} \cdot \nabla) \mathbf{U}_{0}=S u_{3} \mathbf{e}_{x}$. The dynamics that follows from the non-normal system (5) has exponentially decaying vortices that drive spanwise streaks: if $s_{0}$ and $\omega_{0}$ denote the initial amplitudes, then

$$
\begin{aligned}
s(t) & =\left(s_{0}+S \omega_{0} t\right) \mathrm{e}^{-\nu\left(\alpha^{2}+\beta^{2}\right) t} ; \\
\omega(t) & =\omega_{0} \mathrm{e}^{-\nu\left(\alpha^{2}+\beta^{2}\right) t} .
\end{aligned}
$$

Clearly, even if there is no streak initially (i.e., $s_{0}=0$ ), there will be one as time progresses as a consequence of the mixing induced by the downstream vortex. Eventually, however, both will decay. The maximal amplitude of the streak follows from the maximum of $t \exp \left(-\nu\left(\alpha^{2}+\beta^{2}\right) t\right)$, which occurs at a time

$$
t_{\max }=\frac{1}{\nu\left(\alpha^{2}+\beta^{2}\right)} .
$$

Since the maximal amplitude of the downstream component of the streak $\mathbf{u}_{s}$ is $\alpha$, the maximal modulation of the downstream velocity component follows to be

$$
u_{s, \max }=S \frac{\alpha}{\nu\left(\alpha^{2}+\beta^{2}\right)} \omega_{0}
$$

Note that this is proportional to the shear (the larger the shear the stronger the amplification) and to the inverse of the viscosity. This model also allows us to draw conclusions about the sizes of the vortices: Assume that the thickness in the shear direction, i.e., the wave number $\beta$, is given. Then the maximal value of $u_{s, \max }$ is obtained from differentiation of equation (8) for $\alpha=\beta$. If $\beta=\pi / d$, with $d$ the separation between the plates, the wavelength in the spanwise direction is about twice this width, i.e., $\lambda_{\text {span }} \simeq 2 \pi / \beta=2 d$. 


\subsection{Vortex-shear interactions with noise}

Suppose now that we add noise to the above system. Since the two components are already divergence-free, we model the noise by adding the random perturbations $\xi_{s}(t)$ and $\xi_{\omega}(t)$ to each component as follows:

$$
\left(\begin{array}{c}
\dot{s} \\
\dot{\omega}
\end{array}\right)=\left(\begin{array}{cc}
-\lambda & S \\
0 & -\lambda
\end{array}\right)\left(\begin{array}{l}
s \\
\omega
\end{array}\right)+\left(\begin{array}{l}
\xi_{s} \\
\xi_{\omega}
\end{array}\right) .
$$

For simplicity we assume that

$$
\begin{aligned}
\left\langle\xi_{s}(t) \xi_{s}\left(t^{\prime}\right)\right\rangle & =D_{s} \delta\left(t-t^{\prime}\right) \\
\left\langle\xi_{\omega}(t) \xi_{\omega}\left(t^{\prime}\right)\right\rangle & =D_{\omega} \delta\left(t-t^{\prime}\right) \\
\left\langle\xi_{s}(t) \xi_{\omega}\left(t^{\prime}\right)\right\rangle & =0 .
\end{aligned}
$$

The formalism is easily expanded to more general forms. For notational convenience and since the quantitative dependence on wave numbers and viscosity is of minor interest in this section, we have defined $\lambda=\nu\left(\alpha^{2}+\beta^{2}\right)$. If we start at $t=-\infty$, the formal solution is

$$
\begin{aligned}
\omega(t)= & \int_{-\infty}^{t} \mathrm{~d} t^{\prime} \mathrm{e}^{-\lambda\left(t-t^{\prime}\right)} \xi_{\omega}\left(t^{\prime}\right) \\
s(t)= & \int_{-\infty}^{t} \mathrm{~d} t^{\prime} \mathrm{e}^{-\lambda\left(t-t^{\prime}\right)} \xi_{s}\left(t^{\prime}\right) \\
& +S \int_{-\infty}^{t} \mathrm{~d} t^{\prime} \int_{-\infty}^{t^{\prime}} \mathrm{d} t^{\prime \prime} \mathrm{e}^{-\lambda\left(t-t^{\prime}\right)} \mathrm{e}^{-\lambda\left(t^{\prime}-t^{\prime \prime}\right)} \xi_{\omega}\left(t^{\prime \prime}\right) .
\end{aligned}
$$

With this choice of initial time any effects of initial conditions drop out and the effects of noise are highlighted.

\subsection{Correlations}

The equations for the vorticity with their exponential damping and a white-noise source represent an OrnsteinUhlenbeck process and thus lead to exponentially decaying correlations [20,21]. Formally this follows from

$$
\begin{aligned}
C_{\omega, \omega}(t, \tau) & =\langle\omega(t) \omega(\tau)\rangle=\mathrm{e}^{-\lambda(t+\tau)} \\
& \times \int_{-\infty}^{t} \mathrm{~d} t^{\prime} \int_{-\infty}^{\tau} \mathrm{d} \tau^{\prime} \mathrm{e}^{\lambda\left(t^{\prime}+\tau^{\prime}\right)}\left\langle\xi_{\omega}\left(t^{\prime}\right) \xi_{\omega}\left(\tau^{\prime}\right)\right\rangle,
\end{aligned}
$$

which yields, in conjunction with equation (10) and the restriction of the final integral up to $\min (t, \tau)$,

$$
C_{\omega, \omega}(t, \tau)=\frac{D_{\omega}}{2 \lambda} \mathrm{e}^{-\lambda|t-\tau|}
$$

The correlation function for the streaks has two components, one resulting from the noise in the streak components, and one from the non-normal amplification. The cross terms involving the noise sources in both streak and vortex drop out since they are uncorrelated. The streak contribution is again an Ornstein-Uhlenbeck process with an exponentially decaying correlation function as in equation (13). The non-normal amplification gives rise to a contribution from the vortices,

$$
\begin{aligned}
& C_{s, s}^{(\text {vortex })}(t, \tau)= \\
& \int_{-\infty}^{t} \mathrm{~d} t^{\prime} \int_{-\infty}^{t^{\prime}} \mathrm{d} t^{\prime \prime} \int_{-\infty}^{\tau} \mathrm{d} \tau^{\prime} \int_{-\infty}^{\tau^{\prime}} \mathrm{d} \tau^{\prime \prime} S^{2} \mathrm{e}^{-\lambda\left(t-t^{\prime \prime}+\tau-\tau^{\prime \prime}\right)} \\
& \times\left\langle\xi_{\omega}\left(t^{\prime \prime}\right) \xi_{\omega}\left(\tau^{\prime \prime}\right)\right\rangle \cdot
\end{aligned}
$$

Again via equation (10) and the constraint on the domain of integration to $\min \left(t^{\prime}, \tau^{\prime}\right)$, the vortex contribution to the streak-streak correlation function becomes

$$
C_{s, s}^{(\text {vortex })}(t, \tau)=\frac{S^{2} D_{\omega}}{4 \lambda^{3}}(1+\lambda|t-\tau|) \mathrm{e}^{-\lambda|t-\tau|} .
$$

This shows clearly an algebraic contribution that results from the transient-growth characteristic of a non-normal system. For small time-differences, the cusp at the origin for the Ornstein-Uhlenbeck process becomes a rounded, quadratic maximum. The total correlation function

$$
C_{s, s}(t, \tau)=\left(\frac{D_{s}}{2 \lambda}+\frac{S^{2} D_{\omega}}{4 \lambda^{3}}(1+\lambda|\Delta|)\right) \mathrm{e}^{-\lambda|\Delta|},
$$

with the time difference $\Delta \equiv t-\tau$, is symmetric in $\Delta$ and decays monotonically. Formally, this calculation is equivalent to that for a linearly damped process exposed to both white noise (the driving of the streaks) and exponentially correlated noise (the cross coupling from the vortices) $[20,21]$. What is specific to our case is that the coloured-noise component is generated dynamically by the structure of the non-normal system. Cross correlations between vortex and streak can be calculated similarly; and they provide interesting insights into non-normal amplification. We define

$$
C_{\omega, s}(t, \tau)=\langle\omega(t) s(\tau)\rangle
$$

Then, for $t>\tau$, i.e., if the streak is probed before the vortex, cross correlations decay exponentially as

$$
C_{\omega, s}(t, \tau)=\frac{S}{4 \lambda^{2}} D_{\omega} \mathrm{e}^{-\lambda(t-\tau)}, \quad \text { for } t>\tau .
$$

However, for $t<\tau$, i.e., if the streak is probed after the vortex, the driving of the streak by the vortex gives rise to the correlation function

$$
\begin{aligned}
C_{\omega, s}(t, \tau)= & \frac{S}{4 \lambda^{2}} D_{\omega}(1+2 \lambda(\tau-t)) \mathrm{e}^{-\lambda(\tau-t)}, \\
& \text { for } t<\tau,
\end{aligned}
$$

which increases first, for short times, and then decreases. It has a maximum that lies about $21 \%$ above the value at zero, at $\lambda(\tau-t)=1 / 2$. The cross correlation thus provides a convenient and characteristic measure of the lift-up effect. A comparison between the three correlation functions discussed above is shown in Figure 2. 


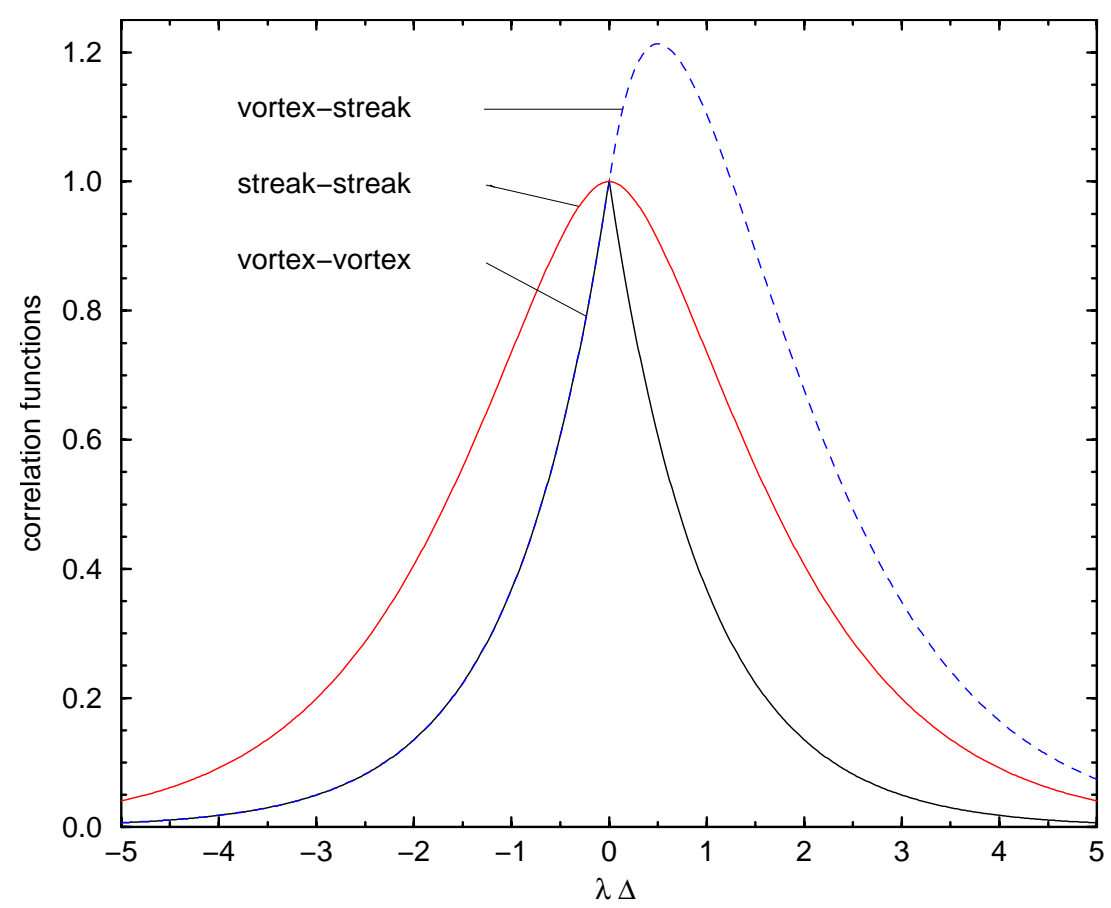

Fig. 2. Correlation functions (see text) for vortex-streak coupling with noise of equal intensity in each component. The correlation functions are normalised by their values at zero, $\Delta$ is the time difference, and $\lambda$, which has dimensions of inverse time, is defined in the text.

\section{Noisy shear flows}

In order to model noise in a shear flow, we have to allow for spatially fluctuating velocity fields. The dynamics we want to study, the interaction between streaks and vortices, is mediated by the background shear flow. While the detailed shape of either object and also the gradient of the shear flow will depend on the specific boundary conditions, the conclusions about the correlation function should be less sensitive. For instance, the vortex-streak recycling mechanism is very similar in flows with rigid boundary conditions and in those with free-slip boundary conditions $[13,14]$. We, therefore, choose the theoretically most convenient form of free slip boundary conditions so that we can use a Fourier representation for the velocity field,

$$
\mathbf{u}(\mathbf{x}, t)=\sum_{\mathbf{k}} \mathbf{u}(\mathbf{k}, t) \mathrm{e}^{\mathrm{ik} \cdot \mathbf{x}}
$$

and also for the noise,

$$
\xi(\mathbf{x}, t)=\sum_{\mathbf{k}} \xi(\mathbf{k}, t) \mathrm{e}^{\mathrm{i} \mathbf{k} \cdot \mathbf{x}}
$$

The linearized Navier-Stokes equation (1) becomes

$$
\dot{\mathbf{u}}(\mathbf{k}, t)+S u_{z}(\mathbf{k}, t) \mathbf{e}_{\mathbf{x}}=-\nu \mathbf{k}^{2} \mathbf{u}(\mathbf{k}, \mathbf{t})+\xi(\mathbf{k}, \mathbf{t}) .
$$

As in the previous section we assume that the wave vectors have no downstream component. The stochastic force $\xi$ (or noise) satisfies

$$
\begin{aligned}
\left\langle\xi_{i}(\mathbf{k}, t)\right\rangle & =0, \\
\left\langle\xi_{i}(\mathbf{k}, t) \xi_{j}\left(\mathbf{k}^{\prime}, t^{\prime}\right)\right\rangle & =A(k) P_{i j}(\mathbf{k}) \delta\left(\mathbf{k}+\mathbf{k}^{\prime}\right) \delta\left(t-t^{\prime}\right),
\end{aligned}
$$

where $A(k)$ is the amplitude of the variance (whose precise functional form does not matter for the moment but which will be discussed further at the end of this section), $P_{i j}(\mathbf{k})=\left(\delta_{i j}-k_{i} k_{j} / k^{2}\right)$ is the transverse projector that enforces the incompressibility condition, and $i$ and $j$ denote Cartesian components. We assume, for simplicity, that the system is infinite; the effects of boundary conditions will be included presently by suitable constraints on $k_{z}$ as in the previous section. The temporal correlation functions we would like to calculate ( $c f$. Sect. 2) are

$$
C_{i j}\left(t, t^{\prime}\right)=\left\langle\left\langle u_{i}(t) u_{j}\left(t^{\prime}\right)\right\rangle\right\rangle
$$

where $\langle\langle\cdots\rangle\rangle$ stands for an integration over all points in the volume under consideration and an average over different realizations of the noise. Given our Fourier representation this becomes

$$
C_{i j}\left(t, t^{\prime}\right)=\sum_{\mathbf{k}}\left\langle u_{i}(\mathbf{k}, t) u_{j}\left(-\mathbf{k}, t^{\prime}\right)\right\rangle ;
$$

now only an average over different realizations of the noise remains for every wave number $\mathbf{k}$. This average can be calculated for each wave number individually and the sum can then be evaluated. Let us first write equation (22) in component form:

$$
\begin{aligned}
\dot{u}_{x}(\mathbf{k}, t)+S u_{z}(\mathbf{k}, t) & =-\nu k^{2} u_{x}(\mathbf{k}, t)+\xi_{x}(\mathbf{k}, t) \\
\dot{u}_{y}(\mathbf{k}, t) & =-\nu k^{2} u_{y}(\mathbf{k}, t)+\xi_{y}(\mathbf{k}, t) ; \\
\dot{u}_{z}(\mathbf{k}, t) & =-\nu k^{2} u_{z}(\mathbf{k}, t)+\xi_{z}(\mathbf{k}, t) .
\end{aligned}
$$

The projection onto divergence-free velocity fields and noise terms implies that the last two equations are coupled; they can thus be represented by a single field $u_{\omega}$ from 
which the $y$ and $z$ Cartesian components can be obtained as follows:

$$
\begin{aligned}
& u_{y}(\mathbf{k}, t)=-u_{\omega}(\mathbf{k}, t) k_{z} / k \\
& u_{z}(\mathbf{k}, t)=u_{\omega}(\mathbf{k}, t) k_{y} / k
\end{aligned}
$$

Now only two equations remain for each $\mathbf{k}$ :

$$
\begin{aligned}
\dot{u}_{x}(\mathbf{k}, t)+\left(S k_{y} / k\right) u_{\omega}(\mathbf{k}, t) & =-\nu k^{2} u_{x}(\mathbf{k}, t)+\xi_{x}(\mathbf{k}, t) \\
\dot{u}_{\omega}(\mathbf{k}, t) & =-\nu k^{2} u_{\omega}(\mathbf{k}, t)+\xi_{\omega}(\mathbf{k}, t) ;
\end{aligned}
$$

and the noise terms satisfy

$$
\left\langle\xi_{a}(\mathbf{k}, t) \xi_{b}\left(\mathbf{k}^{\prime}, t^{\prime}\right)\right\rangle=A(k) \delta_{a b} \delta\left(\mathbf{k}+\mathbf{k}^{\prime}\right) \delta\left(t-t^{\prime}\right),
$$

where the indices $a$ and $b$ stand for, respectively, the noise components in downstream $(x)$ and perpendicular direction (of vortex form, hence index $\omega$ ). Equations $(32,33$ ) are of the same form as equation (9) so we can identify $\lambda$ with $\nu k^{2}$ and $S$ in equation (9) with $S k_{y} / k$ in equation (32). We can therefore use the correlation functions $(13,16)$ and $(18,19)$ calculated previously along with the expansion of the velocity field (25) and (26) to obtain the correlation functions between spanwise and normalvelocity component (subscripts 2 and 3, respectively),

$$
\begin{aligned}
C_{2,2} & =\sum_{\mathbf{k}} \frac{k_{z}^{2}}{k^{2}}\left\langle u_{\omega}(\mathbf{k}, t) u_{\omega}\left(-\mathbf{k}, t^{\prime}\right)\right\rangle, \\
C_{3,3} & =\sum_{\mathbf{k}} \frac{k_{y}^{2}}{k^{2}}\left\langle u_{\omega}(\mathbf{k}, t) u_{\omega}\left(-\mathbf{k}, t^{\prime}\right)\right\rangle, \\
C_{2,3} & =\sum_{\mathbf{k}} \frac{-k_{z} k_{y}}{k^{2}}\left\langle u_{\omega}(\mathbf{k}, t) u_{\omega}\left(-\mathbf{k}, t^{\prime}\right)\right\rangle,
\end{aligned}
$$

in terms of

$$
\left\langle u_{\omega}(\mathbf{k}, t) u_{\omega}\left(-\mathbf{k}, t^{\prime}\right)\right\rangle=\frac{1}{2 \nu k^{2}} A(k) \mathrm{e}^{-\nu k^{2}\left|t-t^{\prime}\right|} .
$$

Thus these correlation functions decay exponentially; and unless there is an asymmetry in the weighting of wave numbers through the amplitude $A(k)$, the cross correlation $C_{2,3}$ vanishes on account of the antisymmetry under reflection of each wave number individually. The correlation function for the downstream component (subscript 1) becomes

$$
\begin{array}{r}
C_{1,1}\left(t, t^{\prime}\right)=\sum_{\mathbf{k}} \frac{A(k)}{2 \nu k^{2}}\left(1+\frac{S^{2} k_{y}^{2}}{2 \nu^{2} k^{6}}\left(1+\nu k^{2}|\Delta|\right)\right) \\
\times \mathrm{e}^{-\nu k^{2}|\Delta|},
\end{array}
$$

where $\Delta=t-t^{\prime}$ is the difference in times. The cross correlations between vortex (as measured by the normal, $i=3$, component of the velocity) and streak (measured by the downstream, $j=1$, component) becomes

$$
\begin{aligned}
C_{3,1}\left(t, t^{\prime}\right)= & \sum_{\mathbf{k}} \frac{S k_{y}^{2}}{\nu k^{3}} \frac{A(k)}{4 \nu k^{2}} \mathrm{e}^{-\nu k^{2}\left(t-t^{\prime}\right)}, \\
& \text { for } t>t^{\prime},
\end{aligned}
$$

and

$$
\begin{aligned}
C_{3,1}\left(t, t^{\prime}\right)= & \sum_{\mathbf{k}} \frac{S k_{y}}{\nu k^{3}} \frac{A(k)}{4 \nu k^{2}}\left(1+2 \nu k^{2}\left(t^{\prime}-t\right)\right) \mathrm{e}^{-\nu k^{2}\left(t^{\prime}-t\right)} \\
& \text { for } t<t^{\prime} .
\end{aligned}
$$

Interchanging 1 and 3 shows the symmetry $C_{1,3}\left(t, t^{\prime}\right)=$ $C_{3,1}\left(t^{\prime}, t\right)$.

The spatial dependence or, equivalently, the wavevector dependence of the correlation functions is given by the summands in equations (35-37). These correlation functions have high powers of the absolute value of $k$ in the denominator, so all expressions are divergent for $k \rightarrow 0$, and thus dominated by the smallest wave numbers. In the absence of shear, this divergence is much milder if we use the form of $A(k)$ appropriate for thermal noise [11]. However, more singular forms have been used for $A(k)$ in an effort to obtain the Kolmogorov $k^{-5 / 3}$ energy spectrum at the level of a one-loop renormalization group [22]. Of course the nonlinear term plays a crucial role in such calculations so it is not clear whether it is appropriate to use such singular forms for $A(k)$ in our study which does not include nonlinearities.

So, unless the form of $A(k)$ favors a different selection, the correlations will be dominated by the smallest wave numbers. Given the bounding surfaces in the $z$-direction, separated by a distance $d$, we have a normal wave number $k_{z}=n \pi / d$, with $n$ a non-vanishing integer, and a continuous spanwise wave number $k_{y}$. The smallest value of $n$ is $n=1$ and the associated optimal $k_{y}$ is $n \pi / d$. Rigid boundary conditions may change these scales somewhat.

\section{Concluding remarks}

We have discussed correlations in noisy shear flow, both at the level of a simple model with two degrees of freedom and in a model with spatially varying velocity fields. The main result is that the non-normal coupling between downstream vortices and streaks is reflected in the temporal cross correlation function between the downstream and normal velocity components.

While there have been many studies of spatial correlations (Townsend [23] summarizes a large body of literature), few have addressed temporal correlations and hardly any the cross correlations we described here. For instance, Farrell et al. [8] study only the variance maintained by forcing a noisy shear flow. Onuki [11] concentrates on the case in which $A(k)$ has the form appropriate for thermal noise and then calculates the renormalisation of the viscosity because of the nonlinear term. Bassam et al. [24] concentrates on energy amplification but not on the correlation functions we consider. Blackwelder and Kovasznay [25] measured the cross correlations but their diagrams do not provide sufficient detail at short times.

It is, therefore, only in recent numerical work on Lagrangian models [15] that the Lagrangian version of this cross correlation has been considered [26]. Analysis of Lagrangian data shows indeed the asymmetry in the cross 
correlation due to the non-normal effects. Eulerian cross correlations have been studied in numerical simulations of turbulent shear flows [27] and they also show the cross correlations and their asymmetry in time [28].

These numerical simulations also indicate that the relation of our linear model with independent, additive and white in time random perturbations to natural fluctuations in a turbulent flow is not as far fetched as it may seem. Certain time scales and quantitative details will be affected by the nonlinearities neglected in our calculation, but if there is an overall shear the main mechanism of non-normal growth will always be present, and that is at the heart of the cross correlations we study here.

Many of the features of the cross correlations we discuss should be accessible experimentally. The exponential correlation in the vortices should be reflected in the correlations of transversal, i.e., spanwise and normal, velocity components. The streaks are a property of the downstream velocity component and the long correlations should be visible there. Finally, the streak-vortex correlations should be noticeable in longitudinal-transversal correlations. We are not aware of experimental measurements of such correlations. The most direct test would involve fluctuations in laminar shear flows. Two experimental set ups come to mind:

1. One could exploit the inevitable free-stream turbulence in a wind tunnel as a noise source. A shear gradient can then be imposed by a flat plate, above which a linear shear gradient forms. Crossed hotwire anemometers can be used to characterise the statistics of the upstream noise and the velocity field above the plate. The free decay of the noise between the point of characterisation and the point of measurement should have a negligible effect. Reynolds numbers should be low enough so that no turbulence transitions in the shear layer are induced.

2. There are several experiments or proposals for driving and influencing flows in electrically conducting fluids by electrical and magnetic fields [29-31]. Random perturbations of well defined strength could be induced by random fluctuations in the currents. Ideally, flow geometries that are closed, such as a Taylor-Couette apparatus, or spatially localized, as in a plane Couette flow $[32,33]$ should be used. Such a setup should allow for a good control of the noise and should avoid complications arising from the advection of perturbations.

Finally, we would like mention that there is growing interest in turbulent transport in magnetohydrodynamics in the presence of a background shear [34]. The techniques developed here can be applied fruitfully to this case as well, as we will show elsewhere [35].

This research was started while we were visiting the Institute of Theoretical Physics, University of California at Santa Barbara, USA, where it was supported in part by the US National Science Foundation under Grant No. PHY94-07194. For further support BE would like to thank the Deutsche Forschungsgemeinschaft and RP the Indo-French Centre for the Promotion of Advanced Research (Project No. 2404-2). We would also like to thank Sriram Ramaswamy for discussions.

\section{References}

1. S. Grossmann, Rev. Mod. Phys. 72, 603 (2000)

2. M. Landahl, J. Fluid Mech. 98, 243 (1980).

3. L. Boberg, U. Brosa, Z. Naturforsch. A 43, 697 (1988)

4. N.L. Trefethen, A. Trefethen, S.C. Reddy, T.A. Driscoll, Science 261, 578 (1992)

5. B.F. Farrell, P.J. Ioannou, Phys. Fluids A 5, 2298 (1993)

6. M.G. Neubert, H. Caswell, Ecology 78, 653 (1997)

7. P.J. Schmid, D.S. Henningson, Stability and transition in shear flows (Springer, New York, 2001)

8. B.F. Farrell, P.J. Ioannou, Phys. Rev. Lett. 72, 1188 (1994)

9. P.J. Ioannou, J. Atmos. Sci. 51, 998 (1995)

10. B.F. Farrell, P.J. Ioannou, Phys. Fluids A 5, 1390 (1993)

11. A. Onuki, Phys. Lett. A 70, 31 (1979)

12. J.M. Hamilton, J. Kim, F. Waleffe, J. Fluid Mech. 287, $317(1995)$

13. F. Waleffe, Phys. Fluids 7, 3060 (1995)

14. F. Waleffe, Phys. Fluids 9, 883 (1997)

15. S. Pope, Turbulent Flows (Cambridge University Press, Cambridge, 2000)

16. S. Nazarenko, N.K.-R. Kevlahan, B. Dubrulle, Physica D 139, 158 (2000)

17. Lord Kelvin, Philos. Mag. 23, 459 (1887)

18. A.D.D. Craik, W.O. Criminale, Proc. R. Soc. (London), A 406, 13 (1986)

19. B. Eckhardt, J. Kronjäger, K. Marzinzik, Nonnormal amplification in viscoelastic fluids, to be submitted

20. H. Risken, The Fokker-Planck Equation (Springer, Berlin, 1996)

21. C.W. Gardiner, Handbook of Stochastic Methods (Springer, Berlin, 1996)

22. See, e.g., A. Sain, Manu, R. Pandit, Phys. Rev. Lett. 81, 4377 (1998) and references therein

23. A.A. Townsend, The structure of turbulent shear flows, 2nd edn. (Cambridge University Press, Cambridge, 1976)

24. B. Bamieh, M. Dahleh, Energy Amplification in Channel Flows with Stochastic Excitation, preprint (1999) (http://online.itp.ucsb.edu/online/hydrot00/bamieh/)

25. R.F. Blackwelder, L.S. Kovasznay, Phys. Fluids 15, 1545 (1972)

26. S.B. Pope, Phys. Fluids 14, 1070 (2002)

27. J. Schumacher, B. Eckhardt, Europhys. Lett. 52, 627 (2000); J. Schumacher, J. Fluid Mech. 441, 109 (2001)

28. B. Eckhardt, J. Schumacher, A. Jachens, Asymmetric time correlations in turbulent shear flows. Proceedings IUTAM meeting on turbulent shear flows, Princeton 2002

29. O. Cardoso, D. Marteau, P. Tabeling, Phys. Rev. E 49, $454(1994)$

30. B.S. Williams, D. Marteau, J.P. Gollub, Phys. Fluids 9, 2061 (1997)

31. U. Brosa, Z. Naturforsch. A 46, 473 (1991)

32. N. Tillmark, P.H. Alfredsson, J. Fluid Mech. 235, 89 (1992)

33. F. Daviaud, J. Hegseth, P. Bergé. Phys. Rev. Lett. 69, $2511(1992)$

34. E.-J. Kim, B. Dubrulle, Phys. Plasmas 8, 813 (2001)

35. B. Eckhardt, R. Pandit, to be published 